\title{
Numerical Investigation in Local Energy Loss Characteristic of U- bend Pipe with Deflector Structure
}

\author{
Zhang Xiaojun a, Yao Qiang ${ }^{b}$, and Miao Yang ${ }^{c}$ \\ College of Mechanical Engineering and Applied Electronics Technology, Beijing University of Technology \\ Beijing 100124, China \\ axjzhang@bjut.edu.cn, ${ }^{\mathrm{b}} 18811016790 @ 163 . c o m,{ }^{\mathrm{c} m i a o y a n g 1985 @ 126 . c o m ~}$
}

Keywords: U-bend; Deflector; Secondary Flow; Local Energy Loss

\begin{abstract}
The problem of local energy loss characteristic induced by the fluid pulsation with high Reynolds number in the U-bend pipe, numerical investigation of the fluid pulsation in the U-bend pipe with different deflector structures were presented with the help of the LES (large eddy simulation). Aimed at four different types of deflector structures, the pressure gradient, secondary flow, eddy and flow resistance were analyzed comparatively. The results show that the bend part in pipe induces the fluid pulsation, and the accelerated or decelerated motion of fluid particles induced by the pressure gradient results in the Dean eddy. During the fluid pulsation, the cores of high velocity eddy and low velocity eddy all draw close to the centerline of pipe, merge together, and form the core zone of steady jet flow. In order to determine the deflector structure, not only the local energy loss but also the flow resistance are need to be considered simultaneously, and the example of Case 4 is presented in this paper.
\end{abstract}

\section{Introduction}

Although the structure of $U$ type pipe is simple, the internal flow is more complicated. The pressure difference of the pipe wall and the comprehensive effect of the centrifugal force will cause the mixing of the flow layer and the collision between the fluid particles. Moreover, it is still a difficult problem to simulate the internal flow state of the pipe flow and control reasonably.

The research of piping flow field is mainly based on the combination of theoretical modeling, numerical analysis and experimental verification. Most of the pipeline research so far is focused on the research of the 90 degree bend and 180 degree bend. Chowdhury R R ${ }^{[1]}$ used the K - epsilon model model to describe the flow law of the turbulent flow field. R. Röhrig S. Jakirlic' and Rütten F ${ }^{[2-3]}$ have shown that the LES model has a good effect on the simulation of eddy in the bend. Wang $\mathrm{S}$ and Yan $\mathrm{B} \mathrm{H}^{[4-5]}$ described the changing process of the eddy. Vaghefi M, Akbari $\mathrm{M}^{[6]}$ simulated the flow field through the 180 degree bend. Compared with the 90 degree bend, 180 degree takes longer distance to restore the stability of the flow field. In order to ensure the stability of the flow field, Dey L, BarbhuiyaA K ${ }^{[7]}$ discussed the effect of the deflector in reducing the fluid impact of bend.

The specialty of the bend structure is the main reason for the unsteady flow filed. In this paper, we aim to stabilize the internal flow field of the piping system with multi-bend structure. LES model is used to describe the internal flow of the system. The method of reasonable layout deflector in the bend is used to control local energy loss. The best piping structure is finally obtained by this method.

\section{Research method}

In order to complete the improvement and optimization of U-bend pipe structure, a numerical model is built on the basis of the finite element method to simulate the motion changes of the fluid transmission process. In LES, large eddies are resolved directly, while small eddies are modeled.

Filter variables are defined as:

$$
\bar{F}(\mathrm{x}, \mathrm{t})=\int_{D} F(x-r, t) H(\mathrm{r}, \Delta) d x^{\prime}
$$


$\mathrm{H}$ is the filter function, $\Delta$ is filter scale, $\mathrm{D}$ is fluid region.

$\mathrm{H}$ is defined as a simulated calculation:

$$
\mathrm{H}(\mathrm{r}, \Delta)=\left\{\begin{array}{c}
1 / V, \Delta \in V \\
0, \text { others }
\end{array}\right.
$$

$\mathrm{V}$ is the volume of the computing grid, The control equation of LES:

$$
\frac{\partial \bar{u}_{i}}{\partial t}+\frac{\partial \bar{u}_{i} \bar{u}_{j}}{\partial x_{i}}=-\frac{1}{\rho} \frac{\partial \bar{P}}{x_{i}}+\vartheta \frac{\partial^{2} \bar{u}_{i}}{\partial x_{j} \partial x_{j}}+\frac{\partial \tau_{i j}}{\partial x_{j}}
$$

Continuity equation:

$$
\frac{\partial \bar{u}_{i}}{\partial \bar{u}_{j}}=0
$$

$\vartheta$ is kinematic viscosity, $\tau_{i j}=\left(\bar{u}_{i} \bar{u}_{j}-\overline{u_{i} u_{j}}\right)$ is sub-grid stress.

The object of this paper is $U$ bend pipe. The flow is $20 \mathrm{~L}$. Work pressure is $1 \mathrm{Mpa}$. The inner diameter is $88 \mathrm{~mm}$. Internal average flow velocity $\left(V_{a}\right)$ is $3.29 \mathrm{~m} / \mathrm{s}$. Turbulence intensity is $4 \%$. Turbulence is medium turbulence intensity. the center curvature radius of three corners is $100 \mathrm{~mm}(\mathrm{R} 1), 105 \mathrm{~mm}(\mathrm{R} 2)$ and $100 \mathrm{~mm}(\mathrm{R} 3)$. The angle between the outlet section and the horizontal plane is 34 degrees. $\mathrm{S}(\mathrm{mm})$ is the length of $\mathrm{AH}$. AH is left wall. The opposite is the right wall. S $(\mathrm{mm})$ is defined as the $\mathrm{X}$ axis to describe the variation of the pressure coefficient and the intensity change of the secondary flow. The specific parameters are shown in Figure1:

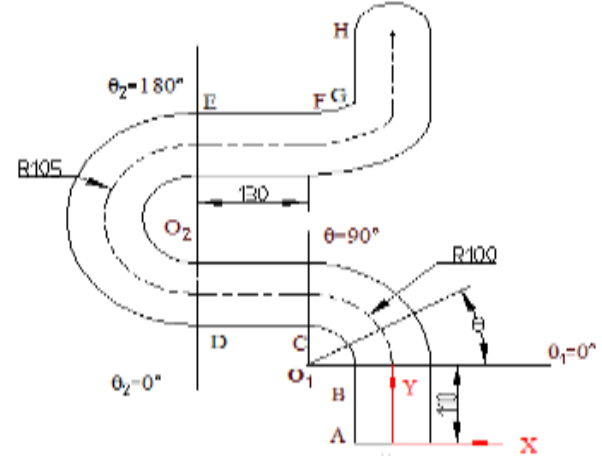

(a) The definition of computational domain and coordinate system

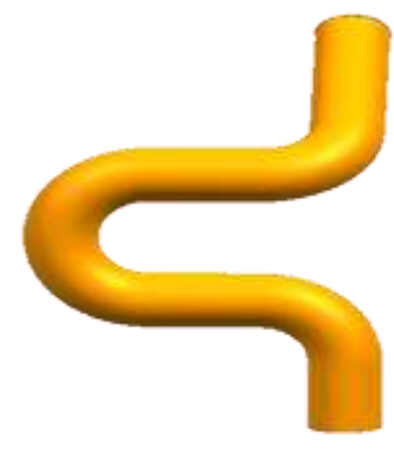

(b) Geometric model

Fig.1 Parameter model structure

The flow field is divided by hexahedral mesh in the Fluent general software environment. The time step is 0.005 seconds. Time step from 700 to 900 is taken as the statistical sample.

This paper describes the flow law of continuous bend flow field. The deflector is used to control the local energy loss. In this study, deflector size and angle is constant. The five cases is obtained by controlling the number and location of the deflector. The thickness of the deflector is $1.2 \mathrm{~mm}$.

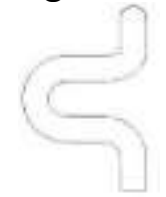

Case1

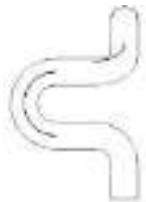

Case2

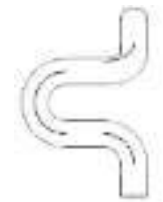

Case3

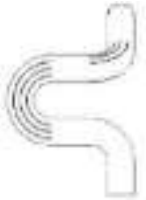

Case4

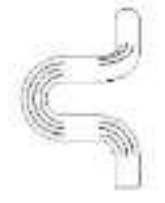

Case5

Fig.2 Design case

\section{Simulation results}

Pressure distribution trend in the first bend of case 2 coincides with case1 pipe. Pressure distribution trend in the next two bends of case 1 coincides with the case 3 (case 4 same argument). In order to make the image clear, figure 3 gives the the pressure distribution law in flow direction of 
the $\mathrm{U}$ pipe, case2, and Case4. The pressure coefficient in the figure is defined as:

$$
C_{P}=\frac{P-P_{0}}{\frac{1}{2} \rho V_{a}^{2}}
$$

$\mathrm{P}_{0}$ is the pressure of the outlet, $V_{a}$ is the average velocity of the pipe internal flow.

In Figure3, the variation trend of the left wall pressure coefficient of the three schemes is both increasing-decreasing-increasing and the variation trend of the pressure coefficient of the right wall is decreasing - increasing - decreasing. At the bend, the positive pressure appears on the outer wall and the negative pressure appears on the inner wall. The pressure difference of the wall in the straight pipe is close to zero. In case 5, the pressure difference at the bend is the lowest. In case 1, the pressure difference at the bend is the highest.

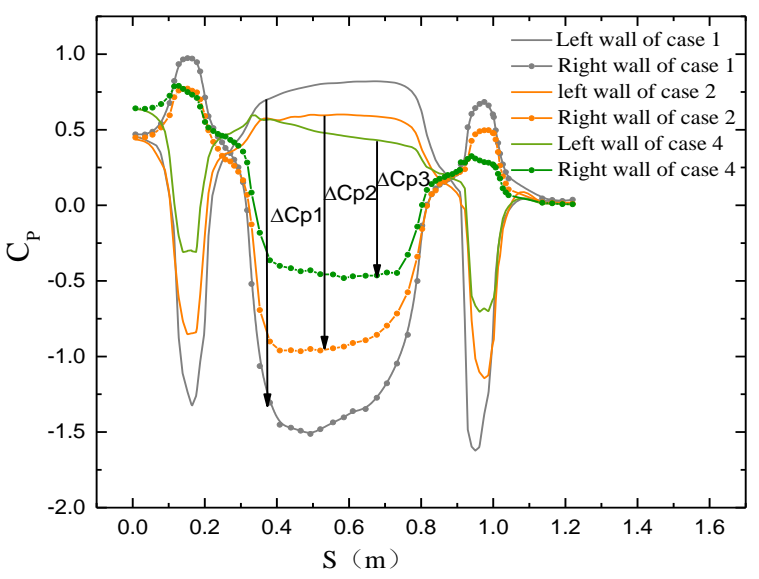

Fig.3 Pressure coefficient distribution of each case

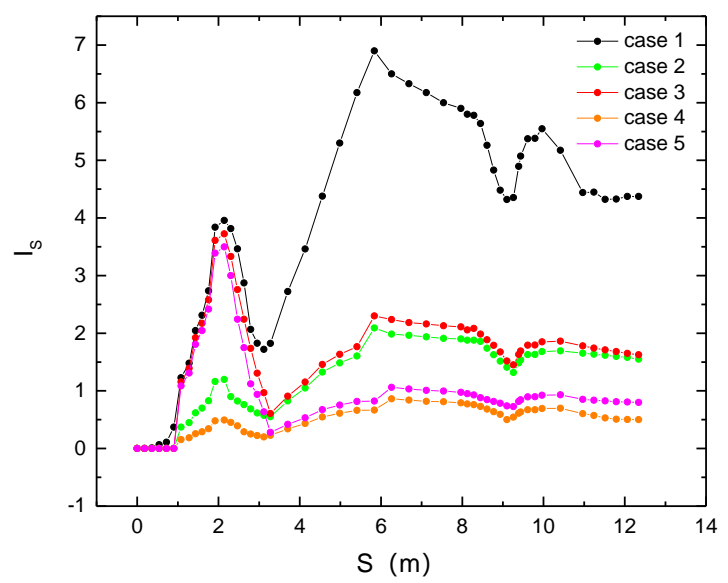

Fig.4 Secondary flow intensity distribution of each case

Figure 4 is intensity distribution of the secondary flow, The A in the figure is defined by the method in below:

$$
I_{s}=\frac{4}{\pi d^{2} V_{a}^{2}} \int_{0}^{2 \pi} \int_{0}^{\frac{d}{2}}\left(U^{2}+V^{2}\right) r d r d \theta
$$

In Figure4, the peak of the secondary flow intensity occurs at the bend. The peak of the 90 degree bend appears at the exit. The 180 degree bend appears in the middle. When the fluid flows through the straight pipe, the trend of the Secondary flow intensity is decreasing. Compared with the secondary intensity of each case, the secondary flow intensity of the case 1 to case 5 is gradually weakened.

Figures 5 are the velocity contour of each case. At the near wall surface, the velocity gradient of each case is large and the change is obvious. From case 1 to case2, there is a movement trend from the wall to the center in the low velocity zone and the high velocity area.
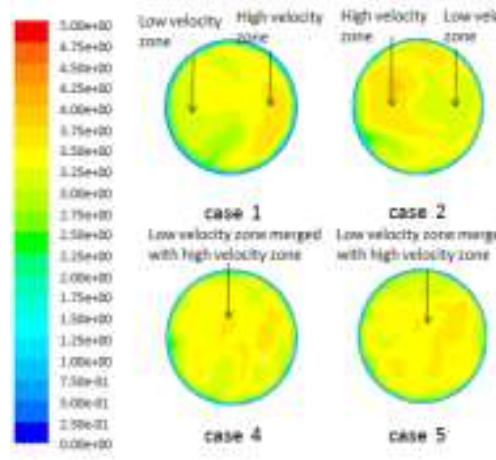

cose 2

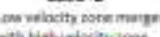

case 3

case 4
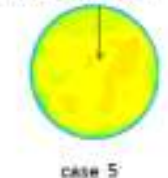

case 5

Fig.5 Export velocity contour of each case

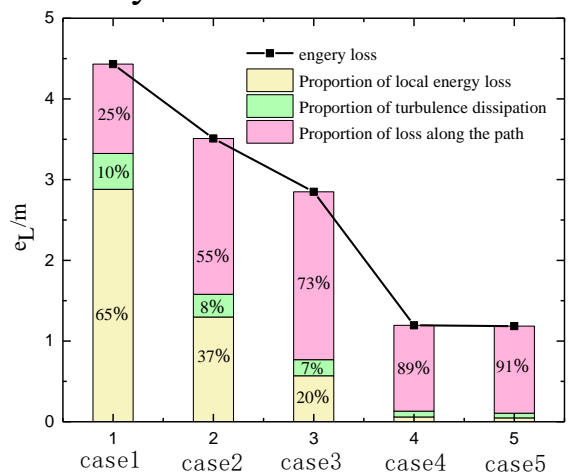

Fig.6 Energy loss of each case 
Figure 6 shows the overall energy loss of each case and the proportion of local energy loss, turbulence dissipation and the linear loss in each case.

The $e_{L}$ in the figure is defined as:

$$
\begin{gathered}
\left(\frac{v^{2}}{2}+\mathrm{gz}+\frac{p}{\rho}\right)_{\text {in }}=\left(\frac{v^{2}}{2}+g z+\frac{p}{\rho}\right)_{o u t}+e_{L} \\
v=\sqrt{\left(U^{2}+V^{2}+W^{2}\right)}
\end{gathered}
$$

The energy loss of case 1 is the highest. The energy loss of case 4 is the lowest.The energy loss gradient from case 4 to case 5 is slow. The proportion of local energy loss shows a downward trend from case 1 to case 5 . The proportion of linear loss shows a growth trend from case 1 to case 5 . The variation trend trend of turbulence dissipation from case 1 to case 5 is slow.

\section{Result analysis}

Figure 3 shows the longitudinal distribution of static pressure that is counted by average time. Because of the effect of curvature radius and centrifugal force, the pressure coefficient of curve shows the trend of increasing first and then decreasing.

In Figure 4 The maximum value of the $90^{\circ}$ bend appears at the exit and $180^{\circ}$ bend appears between $88^{\circ} \sim 92^{\circ}$. The external force is weakened after the fluid flows through the bend and the intensity of the secondary flow is weakened. Because of straight pipe is not enough to make the fluid field to restore stability, the intensity peak of back bend is higher than the previous bend.

Dean eddy is the main form of the secondary flow. Combined with the the trend of the secondary flow intensity of Figure4, We can see that the change of the two flow intensity is closely related to the eddy scale. The intensity change of the secondary flow is the same as the changing trend of the eddy current.In this paper, the velocity in formula (9) is obtained by the formula (8), which is consistent with the actual conditions. In Figure 6, the results of each case show that the local energy loss plays a leading role in the total energy loss. It shows that the control of local energy loss is very important for the optimization of the flow field.

In the above discussion, case 5 shows better results in reducing the pressure difference and the secondary flow eddy scale. In figure5, case 4 and case 5 show the same stable flow field. With the analysis of figure 6 (the comparison of the energy loss of each scheme), the energy loss of case 4 is close to the case5. The deflector has the effect of stabilizing the flow field, but it also brings flow resistance. In this paper, when case 4 continues to add deflector, the effect of the steady flow field will gradually decrease and the flow resistance will also increase.

\section{Conclusions}

1) The bend is the structural factor that causes the unsteady of the flow field. The cyclic variation of eddy generation, enhancement and attenuation is presented in the bend. The deflector can effectively suppress the flow field pulsation and then reduce the energy loss.

2)The acceleration / deceleration of fluid particles caused by pressure gradient is the intrinsic cause of the Dean eddy formation. The size of the eddy reflects the pulsation intensity of the flow field. The pulsation of the large eddy flow field is strong and the small eddy is weak.

3)When the flow field is fluctuating, the low velocity core and high velocity core are easily formed at the exit near the wall. Finally, the stable core jet area is formed.

4)The deflector can reduce the energy dissipation in the fluid transmission and increase the flow resistance, so it is necessary to design a reasonable deflector configuration. In this paper, case4(3 deflectors are arranged at the second and third bend) has a best effect. 


\section{Acknowledgements}

The authors would like to appreciate the support of National Natural Science Foundation of China(No.51605009) and Beijing Natural Science Foundation(No.3174046).

\section{References}

[1] Chowdhury R R, Biswas S, Alam M M, et al. Turbulent flow analysis on bend and downstream of the bend for different curvature ratio[C]// International Conference on Mechanical Engineering: International Conference on Mechanical Engineering. AIP Publishing LLC, 2016:229-239.

[2] R. Röhrig, S. Jakirlić, C. Tropea. Comparative computational study of turbulent flow in a $90^{\circ}$ pipe elbow[J]. International Journal of Heatls\&lsfluid Flow, 2015, 55.

[3] Rütten F, Schröder W, Meinke M. Large-eddy simulation of low frequency oscillations of the Dean vortices in turbulent pipe bend flows[J]. Physics of Fluids, 2005, 17(3):595-112.

[4] Wang S, Ren C, Sun Y, et al. A Study on the Instantaneous Turbulent Flow Field in a 90 Degree Elbow Pipe with Circular Section[J]. Science and Technology and Nuclear Installations,2016, (2016-6-23), 2016, 2016(11):1-8.

[5] Yan B H, Gu H Y, Yu L. CFD analysis of the loss coefficient for a $90^{\circ}$ bend in rolling motion[J]. Progress in Nuclear Energy, 2012, 56:1-6.

[6] Vaghefi M, Akbari M, Fiouz A R. An experimental study of mean and turbulent flow in a 180 degree sharp open channel bend: Secondary flow and bed shear stress[J]. KSCE Journal of Civil Engineering, 2016, 20(4):1582-1593.

[7] Dey L, Barbhuiya A K, Biswas P. Experimental study on bank erosion and protection using submerged vane placed at an optimum angle in a $180^{\circ}$ laboratory channel bend[J]. Geomorphology, 2017, 283:32-40.

[8] Pantokratoras A. Steady laminar flow in a 90 bend[J]. 8,9(2016-9-01), 2016, 8(9). 